\title{
Combining Ordinary Differential Equations with Rigid Body Dynamics: Teach- ing a Second-year Engineering Dynamics Course to Two-year College Grad- uates
}

Dr. Roes Arief Budiman P.Eng., University of Calgary

Received PhD in Materials Science and Engineering at University of Toronto in 2001. Currently a Senior Instructor at University of Calgary and have been teaching Probability \& Statistics for Engineers course in the past three years. Maintain a small research group (1 $\mathrm{PhD}, 1 \mathrm{MEng})$ on pipeline failure and reliability.

Vishash Kumar Sharma 


\title{
COMBINING ORDINARY DIFFERENTIAL EQUATIONS WITH RIGID BODY DYNAMICS: TEACHING A SECOND-YEAR ENGINEERING DYNAMICS COURSE TO TWO-YEAR COLLEGE GRADUATES
}

\begin{abstract}
Students graduating with a two-year technical diploma from vocational colleges are entering a new Energy Engineering Program in Spring 2015 Term at Schulich School of Engineering, University of Calgary. The program provides a path for students with hands-on skills to pursue an engineering bachelor degree. The need to reduce the program completion duration and to provide mathematics courses with sufficient practical aspects has led the School to design a second-year course that combines ordinary differential equations with rigid body dynamics. This course, named ENER 340, has a prerequsite of another course, ENER 240, which combines differential and integral calculus with particle dynamics. We - the ENER 340 instructor team - find that even with such prerequisite course that exposes the students to more elementary concepts of particle dynamics and calculus, students struggled with developing problem solving skills further to solve rigid body dynamics. We find that teaching ordinary differential equations is much easier than teaching rigid body dynamics due to clear logical procedures for solving the former. Students also find it easier to learn the former. We consider several topics that can help blend the two course subjects better based on our teaching experience. We also find that such course blending initiative requires slower teaching and learning speed to allow students to digest the course material better. In addition, a more dedicated textbook that combines both topics is required.
\end{abstract}

\section{Cultures of Learning in Polytechnics and Universities}

A new Energy Engineering Program was launched by Schulich School of Engineering, University of Calgary in 2015 in order to provide a pathway toward a Bachelor of Science (BSc) degree for applicants with a Diploma in Engineering Technology. The applicants normally come from nearby polytechnic colleges (polytechnics), such as Southern Alberta Institute of Technology (SAIT) Polytechnic and Northern Alberta Institute of Technology (NAIT), and have two-year engineering technology diplomas ranging from Mechanical Engineering Technology to Power Engineering Technology.

SAIT Polytechnic states on its Mechanical Engineering Technology program's website [1] that its admission requirements are at least $60 \%$ grades in high school mathematics, physics, and chemistry courses. NAIT states on its Mechanical Engineering Technology program's website [2] that the admission requirements average at about $74 \%$ from these high school courses except chemistry. These admission requirements suggest that some applicants to the Energy Engineering program have lower high school averages than their peers in other engineering programs at the University of Calgary, which typically require at least $85 \%$ average from the same high school courses. It is worth noting that majority of students in these programs, including those at SAIT and NAIT, are either from Calgary or province of Alberta, in which Calgary is located, so that these averages are largely taken from the same pool of students and thus can be compared directly. 
The high school average gap between students in the other engineering programs and applicants of Energy Engineering program reflects a lower academic readiness of the latter group. We believe the academic readiness gap is widened after completing a twoyear diploma program at a polytechnic. Anecdotally, we heard from colleagues on campus that mathematics and physics courses taught at polytechnics focus on using ready-made formulas for a fixed number of problems and do not teach therefore how to analyze a problem using a set of governing equations from scratch. Polytechnic students are thus conditioned in their two-year programs to regard and use mathematics as a formulaic tool that has only one-step process to obtain a solution to a problem. They are not well trained to use mathematics as an analytical tool to translate physical insights, visualize problem geometrically, and make sense of the solution obtained.

The limited use of mathematics to solve problems in polytechnics is widespread [3]; the limited use is actually sensible since the focus of polytechnic education is to deliver "experiential and hands-on" education [4]. Four-year university engineering programs, in contrast, build each of the programs' foundations with first-year mathematics and physics courses that are later used to construct mathematical theories of physical processes relevant for each program. Polytechnic students entering Energy Engineering program therefore have to learn a new culture of learning that relies heavily on mathematical and physical concepts and analyses instead of practical (experiential) and hands-on learning.

This culture of learning difference can be seen from style and depth of textbooks used. The textbook titled Applied Mechanics for Engineering Technology [5] is used for firstyear Engineering Statics in Mechanical Engineering Technology programs in SAIT, while the textbook titled Vector Mechanics for Engineers [6] is used for first-year Engineering Statics in Mechanical Engineering program at the University of Calgary. The Applied Mechanics textbook has little theoretical discussion on applied mechanics, such as the nature of potential energy, and focuses on practical problem solving using free body diagrams. It is concerned with forces (torques) and acceleration (angular accelerations) and doesn't delve into the differential equation structure of the equations of motion. The non-calculus approach of the Applied Mechanics textbook allows the problem solving approach to be presented clearly and logically since all equations involved are algebraic. It is expected that polytechnic students using the Applied Mechanics textbook will gain clarity and learn good problem solving skills - albeit limited - from the non-calculus approach. In fact, the Applied Mechanics textbook is able to combine engineering statics and dynamics in one book due to the non-calculus and focused approach on problem solving.

The Vector Mechanics textbook used in the Mechanical Engineering program, in contrast, uses vector calculus in presenting applied mechanics so that the presentation may seem convoluted and thus do not offer clarity at first glance. Unit vectors in different coordinate systems have to be discussed. It becomes necessary to cover the differential equation structure of the governing equations, and more importantly obtaining solutions become much more complicated than that in the non-calculus approach. One can argue that this complication is required to generalize the formulation so that all mechanics 
problems can be at least expressed mathematically as a set of differential equations. Engineering students taking applied mechanics course may feel dismayed, however, at the difficulty level they face if they ever compare their course content with their polytechnic friends who take practically the same course but learn using the non-calculus approach.

Qualitative comparison of the two textbooks suggests that the level of difficulty of the Applied Mechanics textbook's problems is about $2 / 3$ of the problems' difficulty level given to Mechanical Engineering students in their exams. This decreased difficulty level seems proportional to the high school average gap between students enrolled in polytechnics and those in engineering. The class presentation of applied mechanics using vector calculus and differential equations, however, will increase the difficulty level by at least a factor of two. While exam problems presented to engineering students are $1 / 3$ more difficult, the theory presented to them in class is at least twice more difficult. It is our opinion that engineering students have a much tougher (and possibly more confusing) applied mechanics course. Our teaching experience in engineering mechanics has taught us that the increased course complexity doesn't often translate to much more difficult exam problems because of either exam time constraint, or a lack of solvable problem databank, or the understanding from increased complexity-not just practical, testable skills-is what university should give to students.

Two first-year mathematics courses in SAIT Mechanical Engineering Technology program cover basic algebra and trigonometry, plane analytical geometry, single-variable differential and integral calculus using a textbook titled Basic Technical Mathematics with Calculus [7]. Two first-year mathematics courses in Mechanical Engineering program at University of Calgary start with single-variable differential and integral calculus and end with vector algebra and multivariable calculus using a textbook titled Calculus: A Complete Course [8]. Their comparison reveals that polytechnic students do not learn vector calculus during the first year, which is consistent with the non-calculus approach to applied mechanics. Engineering Technology programs in NAIT and SAIT don't offer a differential equation course which is a mandatory course for any four-year engineering program.

Mathematically, culture of learning in polytechnics differs from that in engineering programs by the absence of courses in vector calculus and differential equation in the former. Vector calculus helps integrate geometry with calculus, hence making formulation of a problem more visual, while differential equation provides a platform to formulate a problem and to test whether its solution under some assumptions is satisfactory when compared with experiments. Polytechnic students are not taught to use mathematics as an analytical tool to translate physical insights, visualize problem geometrically, and make sense of the solution obtained. This topic omission is logical since the students focus on acquiring hands-on skills and therefore use mathematics as a calculation tool. Geometrical and analytical skills normally derived from vector calculus and differential equations will have to be acquired from intuition developed by practice and experience. 


\section{ENER 340 Course Planning and Syllabus}

The course ENER 340 Dynamics for Energy Engineering I is first offered in Summer 2015 term (July-August), as a course in Energy Engineering program at University of Calgary's Schulich School of Engineering. Students graduating from two-year engineering technology diploma programs start taking their Energy Engineering courses in Spring term (May-June), one of them being ENER 240 Introductory Mechanics for Energy Engineering, which is a prerequisite for ENER 340. ENER 240 covers singlevariable calculus and particle dynamics. For example, ENER 240 covers (i) trajectory of a particle moving in air under gravitational force and (ii) friction force. ENER 240 should provide the students with an opportunity to review applied mechanics course(s) they had taken in their two-year diploma programs. Both ENER 240 and 340 are designed with the idea that polytechnic students would be able to learn mathematics better if they are also learning simultaneously their applications.

ENER 340 is delivered in 6 weeks during the Summer term, and each week has 6 hours of lectures and 6 hours of tutorials. The course load per week (pacing) is twice as that in regular Fall (September-December) or Winter (January-April) term. To provide incentives for students to keep up with ENER 340 course progress, weekly quizzes, each worth $6 \%$, and twice weekly tutorial assignments, each worth $1 \%$, are given to students. 6 quizzes and 12 tutorial assignments comprise $48 \%$ of final grade. The continuing assessments by quizzes and tutorial assignments are designed to maintain students' learning appetite given two significant course delivery challenges: (i) twice the speed of regular Fall/Winter term and (ii) two course topics (ordinary differential equations and rigid body dynamics) delivered in one course. Midterm exam's worth is $20 \%$, while final exam's $32 \%$.

To help Energy Engineering students cope with the course load, and in fact throughout the Spring and Summer term loads, Schulich School of Engineering provides a comprehensive tutorial assistance by hiring upper-year Engineering students to work as Learning Assistants in a dedicated, large class room (Devon Academic Resource Centre) where Energy Engineering students can study and seek assistance all day. This learning assistance commitment is at least twice as generous as that offered to other programs and is designed to help the students transition to the new Energy Engineering program.

ENER 340 has the following syllabus for the 6-week long Summer term.

1. Week 1. Ordinary Differential Equations. Review of single variable calculusemphasis on chain rule and definite integral-and particle dynamics. Motivation and applications of ordinary differential equations. Classification of ordinary differential equations (ODE): linear vs. nonlinear, order. Solving differential equations by direct integration. Linear first order ODE. Separable first order ODE. Exact first order ODE.

2. Week 2. Second order ODE. Linear independence and Wronskian. Homogeneous second order ODE with constant coefficients. Linear nonhomogeneous second order ODE. Method of undetermined coefficients. Method of variation of parameters. Euler equation. 
3. Week 3. Power series: Taylor series expansion, convergence, derivative, index shifting. Power series solutions to linear homogeneous second order ODE. Recursive relations. Power series solutions about ordinary points. Hermite's equation and Hermite polynomials.

4. Week 4. Power series solutions about regular singular points. Bessel's equation and Bessel functions. Systems of linear ODE. Method of eigenvalues. Rigid Body Dynamics. System of particles. Centre of mass. Kinetic energy of a system of particles. Work-energy principle; conservation of energy. Impulse and momentum principle; conservation of momentum. Conservation of angular momentum.

5. Week 5. Kinematics of rigid bodies. Velocity analysis. Translation and rotation. Rolling disk. Meshing gears. Acceleration analysis. Normal and tangential acceleration. Kinematics of planar motion.

6. Week 6. Dynamics of rigid bodies. Equation of motion for translational motion. Equation of motion for rotational motion. Moment of inertia.

ENER 340 uses Cengel's Differential Equations for Engineers and Scientists textbook [9] and Beer and Johnston's Vector Mechanics for Engineers textbook [6].

\section{ENER 340 Course Delivery and Student Course Feedback}

The course instructor team consist of the two authors of this paper and three teaching assistants. We took turn teaching the course and have been collaborating on a research project before the ENER 340 teaching assignment. The two-instructor team allowed for better course delivery when compared to our previous teaching experience, but students find our teaching style difference distracting. Their course evaluation feedback states that adjusting to two teaching styles in class and three teaching assistants during tutorials are not conducive to their learning.

We use the course evaluation feedback as a learning assessment for the course in terms of learning barriers they felt, which we think are related to the different cultures of learning in polytechnics and universities. We didn't devise a learning assessment questionnaire due to time constraint; we plan to have such questionnaire when we teach the course again.

There were 49 students taking the course, so there was no concern of student-instructor face-to-face time. Their individual, anonymous course evaluations are summarized below, using keywords that are stated in their course evaluation feedback forms. There are total 82 keyword mentions.

\begin{tabular}{|l|c|c|}
\hline \multicolumn{1}{|c|}{ Comment Keywords } & $\begin{array}{c}\text { Number of times } \\
\text { mentioned }\end{array}$ & Percentage \\
\hline Instruction is good & 3 & $4 \%$ \\
\hline Different instruction styles & 10 & $12 \%$ \\
\hline Not enough time in Summer term & 19 & $23 \%$ \\
\hline Tutorials not effective & 21 & $26 \%$ \\
\hline Teaching assistants not effective & 8 & $9 \%$ \\
\hline Difficult course & 21 & $26 \%$ \\
\hline
\end{tabular}


There are no praises given to the instructors delivering the course, except for the 3 mentions on good instruction. $12 \%$ of total mentions state that the two instructors' different teaching styles become an impediment to learning course material because mainly they had to adjust to different teaching styles within the short 6-week period. We were both always present in class when one of us gave lecture, and we were aware of our teaching style difference and gave feedback to each other during the course duration. This awareness was not reflected in students feedback.

Students do not think tutorial sessions, in which we discussed methods of solving in more details than in class, were effective in giving them assisted time to solve assigned problems. Because we marked weekly quizzes and tutorial assignments given during tutorials, students associated tutorial sessions more with these assessment tools and appeared more stressed out than motivated.

We would like to assume that students would have used learning assistants at the Devon Academic Resource Centre. We visited the Centre several times during the term and often met our students there, numbering 15 to 25 . Their learning capacity to be catalyzed by the Centre seems lower than what we had hoped. Students struggled learning the course material, and we believe this is a reflection of the learning culture they acquired at polytechnics, where they used mathematics as a calculation tool.

We were asked by 2-4 students during class, who asked why they had to learn differential equations. When we stated that differential equation is what we use to predict what's going to happen in a mechanical system, they were rather mystified by the power of mathematics to make predictions. This question-and-answer experience is anecdotal, but it carries an important lesson that changing their perspective may be needed to convince the students to learn mathematics more persistently and thoroughly than what they initially thought or were taught in polytechnics.

This change of perspective is, in our opinion, crucial since it will convince them that the huge time investment they undertake when learning differential equations will be rewarded with improved understanding-theoretical and practical-of mechanical systems they have learned by practice. This perspective change will also lessen their resistance on the necessity to present rigid body dynamics in a much more complicated fashion than what they had used to in polytechnics. The difficulty they feel-indicated by the $26 \%$ mentions-is related to their academic readiness that stresses more in using mathematics not as an analysis tool.

The combined material of differential equation and rigid body dynamics taught in a 6week term is admittedly tough for any student to take. The course material is, however, a prerequisite for many courses students subsequently have to take in Fall and Winter term. It would be difficult, if not impossible, to move the course to either Fall or Winter term in order to have a 13-week duration, as it would produce a longer program completion time. However, $23 \%$ of the mentions are precisely about the lack of learning time when the course is delivered in 6-week Summer term. 


\section{Lessons Learned}

Given that ENER 340 has to be delivered in a 6-week period, we suggest that the course material be either (i) reduced to its bare minimum or (ii) focused on either differential equation or rigid body dynamics. Providing a calculus-refresher short course to the students seems necessary, and this short course would prepare students better for differential equations. The prerequisite ENER 240 might have to emphasize developing geometrical visualization skills using differential calculus. ENER 340 should devote more instruction time in differential calculus than integral calculus.

Removing two topics: (i) power series solutions to differential equations and (ii) system of particle would represent a bare minimum course in differential equation and rigid body dynamics. This removal might help the students learn ENER 340 better. The original course syllabus would be minimized into

1. Week 1. Ordinary Differential Equations. Review of single variable calculusemphasis on chain rule and definite integral-and particle dynamics. Motivation and applications of ordinary differential equations. Solving differential equations by direct integration.

2. Week 2. Linear first order ODE. Separable first order ODE. Exact first order ODE. Second order ODE. Linear independence and Wronskian. Homogeneous second order ODE with constant coefficients.

3. Week 3. Linear nonhomogeneous second order ODE. Method of undetermined coefficients. Method of variation of parameters.

4. Week 4. Rigid Body Dynamics. Kinematics of rigid bodies. Velocity analysis. Translation and rotation. Rolling disk. Meshing gears.

5. Week 5. Acceleration analysis. Normal and tangential acceleration. Kinematics of planar motion.

6. Week 6. Dynamics of rigid bodies. Equation of motion for translational motion. Equation of motion for rotational motion. Moment of inertia.

The rigid body dynamics part will resemble the applied mechanics course they have taken in polytechnics, but the differential equation part will instill connections between differential equations and equations of motion for rigid body dynamics. The conservation laws could be removed in order to focus more on problem solving skills using free body diagrams.

Assessment tools have to be reduced to a minimum to allow students to independently develop problem solving skills. We find an increased number of assessment tools counterproductive since students are more stressed out than motivated when attending tutorials. Our initial goal to maintaining students' learning appetite using weekly quizzes and twice weekly tutorial assignments didn't work as $26 \%$ of the mentions state that tutorials are not effective.

We find that teaching ODE is easier than rigid body dynamics because ODE provides clear systematic paths to classifying and solving differential equations. Students find it difficult to learn differential equation, but once they understand classification scheme and 
methods of solution they could rigidly apply this procedure to obtain a solution. Rigid body dynamics has no such systematic solution paths. Governing equations of mechanics are not many, but they are intimately related to underlying physical concepts that require vector calculus. A lack of basic vector calculus background, such as understanding in unit vectors and coordinate systems, reduces students' ability to connect the concise equations of rigid body motion with geometry. Students often don't know how to convert problem's statements into a set of equations because the connection between geometry and differential calculus was emphasized when they were in polytechnics.

A lack of systematic pathways to solving rigid body problems-or in fact to solving all mechanics problems-complicates our effort in combining differential equations with rigid body dynamics. Our goal to connect them through this course was not successful. Students are told that they are connected; historically we might tell them that it was exactly what Isaac Newton achieved, but they might not fully appreciate the connection.

Students' varied backgrounds, ranging from Mechanical Engineering Technology to Power Engineering Technology, do not help our course delivery. Some students complain that students with Mechanical Engineering Technology diploma understand the course material better. More class practice in solving problems can lessen the background disparity, which suggests that more tutorial times per week are needed. Currently, 6 hours of tutorials per week are prescribed for ENER 340. The increased tutorial times might mean incorporating the Devon Academic Resource Centre's study time into ENER 340's tutorial times.

The two-instructor team we had doesn't help students learn since they have to adjust to different teaching styles in a 6-week term. Although a course combining two different topics such as ENER 340 may convince some engineering schools to have two (or more) instructors due to their expertise, one instructor in our opinion is better to maintain continuity in mathematical notations and teaching style. It might be our folly to have thought that having more than one instructor helps plan and deliver course better, but we have forgotten that classroom teaching is akin to stand-up performance where the audience (students) need to get acquinted well with its actor and storyline.

We also think that a dedicated course textbook is required. The textbook would connect differential equation and rigid body dynamics seamlessly, not to mention achieving consistent mathematical notations; such textbook is not yet available. Our two textbooks suffer from different notations and unnecessary details for each topic. We didn't use teaching tools such as a simple gyroscope to discuss angular momentum or flexible linkages to discuss centre of mass. Such tools may prove useful to instill the practical aspects of mechanics despite increased mathematical complexity.

\section{Acknowledgments}

We would like to thank Professors Bill Rosehart and Anders Nygren of Schulich School of Engineering, University of Calgary, for weekly meetings in Summer 2015, where many discussions on ENER 340 teaching pedagogy, that motivated this paper, took place. 


\section{References}

1. http://www.sait.ca/programs-and-courses/full-time-studies/diplomas/mechanical-engineeringtechnology-course-overview/admission-and-selection.php. Accessed on January 18th, 2016.

2. http://www.nait.ca/77649.htm. Accessed on January 18th, 2016.

3. H. Zhao, "Improve the vocational college students' interest in learning my optimizing the teaching contents of advanced mathematics," International Conference on Information Systems and Computing Technology, Sep 15-16, 2013; Information Systems and Computing Technology, L. Zhang and Y. Gu, eds., 103-106 (2013).

4. http://www.nait.ca/96800.htm. Accessed on January 19th, 2016.

5. K. M. Walker, Applied Mechanics for Engineering Technology (Prentice-Hall, 8th Edition, 2008).

6. F. P. Beer, E. R. Johnston, Jr., and W. E. Clausen, Vector Mechanics for Engineers (McGraw-Hill, 7th Edition, 2004).

7. A. J. Washington, Basic Technical Mathematics with Calculus (Pearson, 10th Edition, 2014).

8. R. A. Adams and C. Essex, Calculus: A Complete Course (Pearson, 8th Edition, 2013).

9. Y. Cengel and W. J. Palm III, Differential Equations for Engineers and Scientists (McGraw-Hill, First Edition, 2013). 